\title{
Alternative Donor-Acceptor Stacks from Crown Ethers and Naphthalene Diimide Derivatives: Rapid, Selective Formation from Solution and Solid State Grinding
}

Gayane Koshkakaryan, ${ }^{\dagger}$ Liana M. Klivansky, ${ }^{\dagger}$ Dennis Cao, ${ }^{\dagger}$ Marian Snauko, ${ }^{\dagger}$ Simon Teat, ${ }^{\ddagger}$ Jochem O. Struppe, ${ }^{\#}$ Yi Liu* ${ }^{\dagger}$

The Molecular Foundry, Lawrence Berkeley National Laboratory, Berkeley, CA 94720, USA

Self assembling $\pi$-conjugated molecules into ordered structures are of increasing interest in the field of organic electronics. ${ }^{1}$ One particular example is charge transfer complexes containing columnar alternative donor-acceptor (ADA) stacks, ${ }^{2}$ where neutral and ionic ground states ${ }^{3}$ can be readily tuned to modulate electrical, optical, and ferroelectrical properties. ${ }^{4}$ Aromaticaromatic and charge transfer interactions ${ }^{5}$ have been the leading driving forces in assisting the self-assembly of ADA stacks. Various folding structures containing ADA stacks were assembled in solution with the aid of solvophobic $^{6}$ or ion-binding interactions. ${ }^{7}$ Meanwhile, examples of solid ADA stacks, which are more appealing for practical use in devices, were obtained from cocrystalization of binary components ${ }^{8}$ or mesophase assembly of liquid crystals in bulk blends. ${ }^{9}$ Regardless of these examples, faster and more controllable approaches towards precise supramolecular order in the solid state are still highly desirable.

Donor-acceptor preorganization in 1:1 inclusion complexes ${ }^{10}$ formed between a macrocyclic host and a guest is advantageous in achieving ordered stacking, despite that only a minimum number of donor and acceptor units is involved. Extending the stacking is possible by incorporating a second bridging guest, while only scarce success was demonstrated ${ }^{11}$ through delicate cocrystalization processes. Here we report the rapid and selective assembly of ADA stacks from an electron rich crown ether and electron deficient naphthalene diimide (NDI) derivatives, (Scheme 1) either through a solution process (within minutes) or from mechanical grinding of the binary mixture. Single crystal and powder X-ray diffraction (PXRD) analyses provided unambiguous structural information, while cross polarization magic angle spinning (CPMAS) ${ }^{15} \mathrm{~N}$ and ${ }^{13} \mathrm{C}$ NMR spectra presented further information on solid state chemical environment.
Scheme 1. Formation of 1:2 complex from crown ether 1 $(1.0 \mathrm{~m} M)$ and NDI $2(2.0 \mathrm{~m} M)$.



(DNP) containing crown ether $1(60 \mu \mathrm{mol})$ in a refluxing MeCN solution of NDI derivative 2a (120 $\mu \mathrm{mol}, 2.0 \mathrm{~m} M$ ) resulted in a clear red solution. Upon cooling to room temperature, a purple precipitate formed quickly, which was shown to contain $\mathbf{1}$ and $\mathbf{2 a}$ in a $1: 2$ ratio by both ${ }^{1} \mathrm{H}$ NMR spectroscopy and elemental analysis (see Supporting Information). This composition implied the formation of ADA stack 3a where 2a molecules were arranged inside and outside the crown cavity respectively to extend the donoracceptor interactions. Notably, 3a was formed within minutes in $98 \%$ yield from high-dilution conditions $(1.0 \mathrm{mM})$, indicating that the selfassembly is thermodynamically highly favored.

Synchrotron single crystal X-ray analysis confirmed $^{12}$ the ordered ADA stacking. One NDI unit was positioned (Figure 1a) centersymmetrically in the cavity of crown ether, in an orientation to maximize its overlap with two surrounding DNP ring systems. An outside NDI unit aligned eclipsed to its included counterpart. All the aromatic cores were arranged face-centered along the crystallographic $b$ axis, with a face-to-face distance around $3.4 \AA$. $\pi$ Stacking columns were arranged parallelly along $a$ - and $c$-axis (Figure 1b). The face-centered ADA arrangement is reminiscent of the packing in covalently linked donor-acceptor [2] catenanes. ${ }^{10 \mathrm{c}}$ No intramolecular hydrogen bonding interactions were identified. 

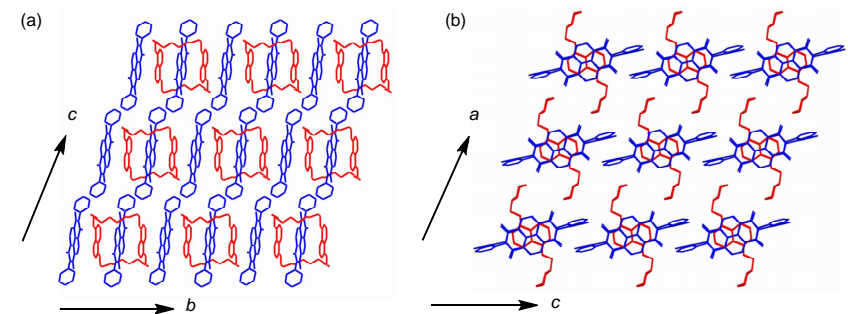

Figure 1. Wireframe representation of X-ray structure of 3a, viewing along (a) the crystallographic $a$ axis, and (b) the $b$ axis. NDI units were colored in blue and the crown ether in red. Solvent molecules and hydrogen atoms were omitted for clarity.

CPMAS NMR experiments provided further information on the chemical environment in the solid state. Surprisingly, the seemingly different "inside" and "alongside" $\mathbf{2 a}$ moiety in the complex 3a were situated in indistinguishable environment, as illustrated by solid state ${ }^{15} \mathrm{~N}$ NMR spectroscopy. (See Figure S1a in Supporting Information) Only two resonances, corresponding to the pyridyl and diimido $\mathrm{N}$ atoms, were observed in the ${ }^{15} \mathrm{~N}$ NMR spectrum of 3a. In comparison, the spectrum of $\mathbf{2 a}$ also contained two resonances that displayed slight shifts. While the peak shifting could be explained by shielding effect imposed by adjacent DNP ring systems, the degeneracy in 3a suggested that the polyethylene glycol chains on the macrocycle had little effect on the chemical environment of the included acceptor. Meanwhile, ${ }^{13} \mathrm{C}$ NMR spectrum of 3a (See Figure S1b in Supporting Information) appeared to be the sum of $\mathbf{1}$ and $\mathbf{2 a}$ except some noticeable peak shifting and splitting, consistent with the observations from ${ }^{15}$ N NMR spectroscopy.

The high affinity of $\mathbf{2 a}$ towards $\mathbf{1}$ was distinct from other NDI derivatives that were known to give conventional $1: 1$ inclusion complex rather than the elusive $1: 2$ complex. ${ }^{10 c, d}$ Indeed, a survey of an array of NDI derivatives $\mathbf{2 a}-\mathbf{2 h}$ (Scheme 1) revealed that only $\mathbf{2 a}$ and $\mathbf{2 b}$ led to ADA stacks while $\mathbf{2 c - 2 h}$ gave 1:1 inclusion complexes that remained in solution. ${ }^{10 \mathrm{c}}$ Increase or decrease of electron deficiency in structurally similar $\mathbf{2 c}$ and $\mathbf{2 d}$ had an adverse effect on ADA stack formation. ${ }^{13}$ In addition, in a competition experiment where $\mathbf{2 a}, \mathbf{1}$ and the better $\pi$-acceptor, paraquat hexafluorophosphate, were mixed in $\mathrm{CD}_{3} \mathrm{CN}$, 3a was formed and precipitated exclusively. ${ }^{14}$ Although detailed mechanistic investigations were needed, these results suggested that the selective formation of 1:2 ADA stack over 1:1 complex was not directly correlated to the acceptor's electron deficiency.

Moreover, the assembly was tested under solid-to-solid mechanical grinding conditions. Upon grinding a 1:2 mixture of $\mathbf{1}$ and $\mathbf{2 a}$ using mortar and pestle in the presence of a few drops of $\mathrm{MeCN},{ }^{15}$ a purple color quickly developed, (inset in Figure 2) consistent with donor-acceptor charge-transfer interactions. ${ }^{16}$ PXRD analysis of the resulting solid after 10 min grinding revealed (Figure 2) a pattern virtually identical to that of the solution grown crystalline sample, concurrent with the absence of diffractions of individual components. It was noteworthy ${ }^{17}$ that very few examples of inclusion complexes had been obtained through mechanical grinding. It was demonstrated here that high degree of organization, which involved both threading and stacking in the case of ADA stacks, were made possible by simple mechanical grinding.

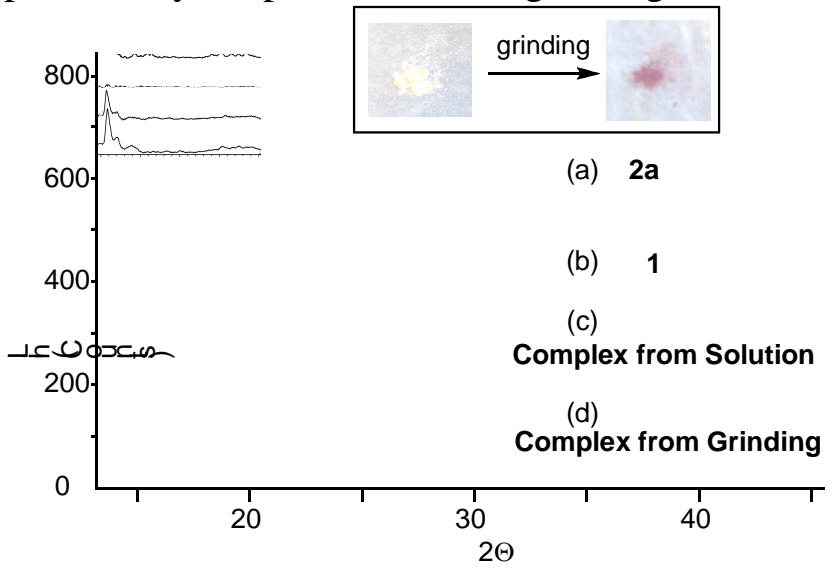

Figure 2. PXRD spectra of (a) 2a, (b) 1, (c) complex 3a from solution process, and (d) complex obtained from mechanical grinding. The inset shows the color change from offwhite to purple after grinding.

In summary, we have shown a unique host-guest system that rapidly leads to extended 1D ADA stack. The 1:2 complex can be obtained as a precipitate from solution within minutes or, more remarkably, from a solid-to-solid mechanical grinding process. The ADA stacking was confirmed by single crystal and powder $\mathrm{XRD}$ analysis and further characterized by solid state CPMAS ${ }^{13} \mathrm{C}$ and ${ }^{15} \mathrm{~N}$ NMR spectroscopy. Current findings not only provide a convenient way to a novel class of ADA stacks involving macrocyclic host, but also represent an important step in transferring electro-active host-guest systems from solution to the solid state. 
Investigations on their electronic and photophysical properties are currently underway. In addition, the precise alignment of functional groups, such as the reactive pyridyl units, sets the stage for covalent bond formation towards interlocked structures.

Acknowledgement. This work was supported by the Office of Science, Office of Basic Energy Sciences, of the U. S. Department of Energy under contract No. DE-AC02-05 CH11231.

$\dagger$ The Molecular Foundry, Lawrence Berkeley National Laboratory, CA, US.

¥ Advanced Light Source, Lawrence Berkeley National Laboratory, CA, US.

${ }^{\#}$ Bruker BioSpin Corp., Billerica, MA, US.

\section{References}

(1) Forrest, S. R.; Thompson, M. E. Chem. Rev. 2007, 107, 923-925.

(2) M. Schwoerer, H. C. Wolf Organic Molecular Solids 2007, Wiley-VCH, Weinheim.

(3) (a) McConnell, H. M.; Hoffman, B. M.; Metzger, R. M. Proc. Natl. Acad. Sci. U.S.A. 1965, 53, 46-50. (b) Torrance, J. B.; Vazquez, J. E.; Mayerle, J. J.; Lee, V. Y. Phys. Rev. Lett. 1981, 46, 253-257.

(4) (a) Okamoto, H.; Mitani, T.; Tokura, Y.; Koshihara, S.; Komatsu, T.; Iwasa, Y.; Koda, T.; Saito, G. Phys. Rev. B. 1991, 43, 8224-8232. (b) Collet, E. et al. Science 2003, 300, 612-615. (c) Horiuchi, S.; Tokura Y. Nature Mater 2008, 7, 357-366.

(5) (a) Hunter, C. A.; Sanders, J. K. M. J. J. Am. Chem. Soc. 1990, 112, 5525-5534. (b) Hunter, C. A.; Lawson, K. R.; Perkins, J.; Urch, C. J. J. Chem. Soc., Perkin Trans. 2 2001, 3, 651-659. (c) Griffiths, K. E.; Stoddart, J. F. Pure. Appl. Chem. 2008, 80, 485-506.

(6) (a) Lokey, R. S.; Iverson, B. L. Nature 1995, 375, 303-310. (b) Gabriel, G. J.; Iverson, B. L. J. Am. Chem. Soc. 2002, 124, 15174-15175. (c) Gabriel, G. J.; Sorey, S.; Iverson, B. L. J. Am. Chem. Soc. 2005, 127, 2637-2640. (d) Zhou, QZ.; Jiang, X-K.; Shao, X-B.; Chen, G-J.; Jia, MX.; Li, Z-T. Org. Lett. 2003, 5, 1955-1958.

(7) (a) Ghosh, S.; Ramakrishnan, S. Angew. Chem. Int. Ed. Engl. 2004, 43, 3265-3268. (b) Ghosh, S.; Ramakrisham, S. Angew. Chem. Int. Ed. 2005, 44, 5441-5447.
(8) (a) Patrick, C. R.; Prosser, G. S. Nature 1960, 187, 1021-1021. (b) Hamilton, D. G.; Lynch, D. E.; Byriel, K. A.; Kennard, C. H. L. Aust. J. Chem. 1997, 50, 439-446.

(9) (a) Percec, V. et al. Nature 2002, 419, 384387. (b) Park, L. Y.; Hamilton, D. G.; McGehee, E. A.; McMenimen, K. A. J. Am. Chem. Soc. 2003, 125, 10586-10590. (c) Reczek, J. J.; Villazor, K. R.; Lynch, V.; Swager, T. M.; Iverson, B. L. J. Am. Chem. Soc. 2006, 128, 7995-8002. (d) Pisula, W.; Kastler, M.; Wasserfallen, D.; Robertson, J. W. F.; Nolde, F.; Kohl, C.; Mullen, K. Angew. Chem. Int. Ed. 2006, 45, 819-823.

(10) (a) Stoddart, J. F. Pure. Appl. Chem. 1988, 60, 467-472. (b) Odell, B.; Reddington, M. V.; Slawin, A. M. Z.; Spencer, N.; Stoddart, J. F.; Williams, D. J. Angew. Chem. Int. Ed. Engl. 1988, 27, 1547-1550. (c) Hamilton, D. G.; Davies, J. E.; Prodi, L.; Sanders, J. K. M. Chem. Eur. J. 1998, 4, 608-617. (d) Kaiser, G.; Jarrosson, T.; Otto, S.; Ng, Y-F.; Bond, A. D.; Sanders, J. K. M. Angew. Chem. Int. Ed. 2004, 43, 1959-1962.

(11) (a) Ortholand, J-Y.; Slawin, A. M. Z.; Spencer, N.; Stoddart, J. F.; Williams, D. J. Angew. Chem. Int. Ed. Engl. 1989, 28, 13941395. (b) Asakawa, M.; Dehaen, W.; L'abbé, G.; Menzer, S.; Nouwen, J.; Raymo, F. M.; Stoddart, J. F.; Williams, D. J. J. Org. Chem. 1996, 61, 9591-9595.

(12) Crystals suitable for X-ray single crystal analysis were grown by a heterogeneous layer diffusion method. It is noteworthy that although the stack required a 1:2 ratio of two components, the assembly proved to be effective starting from exceedingly biased concentrations. See Supporting Information for crystallographic data and crystal growing conditions.

(13) The electrostatic potential (ESP) plots of 2a2d revealed (See Supporting Information) that 2c had the most positive $\pi$ clouds, while $\mathbf{2 d}$ had the least.

(14) See Supporting Information for a detailed description of the competition experiment.

(15) The presence of solvent can significantly shorten the grinding time. A ball mill apparatus worked equally efficiently as mortar-and-pestle grinding. 
(16) The purple color is a good indication for the ADA stack formation, which is absent when mixtures of $\mathbf{1}$ and $\mathbf{2 c - h}$ are grinded.

(17) (a) Orita, A.; Okano, J.; Tawa, Y.; Liang, L.; Otera, J. Angew. Chem. Int. Ed. 2004, 43, 37243728. (b) Kihara, N.; Hinoue, K.; Takata, T. Macromolecules 2005, 38, 223-226. (c) Hsueh, S.-Y.; Cheng, K.-W.; Lai, C-C.; Chiu, S.-H. Angew. Chem. Int. Ed. 2008, 47, 4436-4439.

This work was supported by the Office of Science, Office of Basic Energy Sciences, of the U.S. Department of Energy Under Contract No. DE-AC02-05CH11231. 
ABSTRACT: The atypical 1:2 complexation between an electron rich crown ether host and electron deficient naphthalene diimide-based guests led to the formation of alternative donor-acceptor (ADA) stacks. The ADA stacks can be expediently obtained in high yield as polycrystalline aggregates from solution. More remarkably, the high degree of organization has also been realized in a simple solid-tosolid mechanical grinding process. The solid-state structures have been verified by solid-state NMR spectroscopy, single crystal and powder X-ray diffraction analysis. The current findings not only provide convenient ways of obtaining novel donor-acceptor stacks involving a macrocyclic host, but also represent an important step in transferring electro-active host-guest systems from solution to the solid state. 